\title{
First Report of Fulminant Budd- Chiari Syndrome Treated by Direct Intrahepatic Portocaval Shunt in Vietnam
}

\author{
Thai Van Nguyen ${ }^{a} \quad$ Thinh Tien Nguyen ${ }^{a}$ Hoang Duc Dong ${ }^{b}$ \\ Huy Quang Duong ${ }^{c}$ \\ aDepartment of Hepato-Gastroenterology, 108 Military Central Hospital, Hanoi, Vietnam; \\ bepartment of Internal Medicine, Thai Nguyen University of Medicine and Pharmacy, \\ Thainguyen, Vietnam; 'Department of Gastroenterology and Hepatology, Viet Nam Military \\ Medical University, Hanoi, Vietnam
}

\section{Keywords}

Budd-Chiari syndrome - Direct intrahepatic portosystemic shunt - Fulminant hepatic failure

\section{Abstract}

Budd-Chiari syndrome (BCS) is a rare disorder caused by hepatic venous outflow obstruction that can lead to acute liver failure proposing liver transplantation or transjugular intrahepatic portosystemic shunt. However, the transjugular intrahepatic portosystemic shunt is not always successful due to the entire hepatic vein thrombosis while transplantation is not unfailingly feasible. In these situations, the direct intrahepatic portosystemic shunt (DIPS) is a viable alternative that may ameliorate portal hypertension in these patients. We described a case of a 21-year-old male with fulminant hepatic failure owning to BCS with a 4-day history of abdominal pain and nausea. Laboratory workup, including viral, autoimmune etiologies JAK2 mutation, Factor $V$ Leiden, antiphospholipid antibody syndrome, was negative. The patient's clinical status worsened with hepatic encephalopathy stage II despite administering anticoagulation. Thus, the patient underwent urgently DIPS after unaccessible to the creation of a transjugular intrahepatic portosystemic shunt and impossible to transplantation. The patient's health was improved and discharged. Fulminant Budd-Chiari is a rare disease to be demanding prompt treatment. While transplantation or transjugular intrahepatic portosystemic shunt is failed, the DIPS is considered an alternative candidate associated with clinical improvement.

\section{Karger"}


Nguyen et al.: DIPS for Fulminant BCS

\section{Introduction}

Budd-Chiari syndrome (BCS) was described by George Budd and Hans Chiari [1], which is associated with hepatic venous outflow tract obstruction by thrombosis or structural compression at the level of the main hepatic vein (HV) or the extrahepatic segment of the inferior vena cava (IVC) [2]. The clinical manifestation of BCS may be asymptomatic until cause acute liver failure. Three classic clinical features include abdominal pain, ascites, and hepatomegaly. The complication of BCS depends on the level and rapidity of venous obstruction [3]. The TIPS has been proposed to use early in selected cases because of associatively high mortality from BCS patients who underwent medical therapy alone. In terms of fulminant/acute liver failure, the patient is in urgent need of TIPS and considered early liver transplant in suitable candidates if BCS-TIPS score $>7$. However, in some case, the HV is completed obstruction, TIPS does not perform whereas transplantation seems an improbability; direct intrahepatic portosystemic shunt (DIPS) is rescue of the patient from fatal conditions. The DIPS connects the main portal vein or one of its branches directly to the IVC instead of one of the hepatic branches. In any case, long-term anticoagulation therapy with warfarin is recommended in those with a demonstrable hypercoagulable state [4]. Herein is introduced a young male with fulminant BCS was successfully treated with DIPS creation.

\section{Case Presentation}

A 21-year-old male was admitted to our hospital as a transfer from another hospital to receive a high level of care due to ascites, abdominal pain, and nausea for 4 days. The patient and his family had no previous disease. On admission, he complained of dyspnea, abdominal distention with the presence of shifting dullness. The patient's workup was consistent with decompensated liver cirrhosis. His vital signs were as follows: Glasgow Coma Scale score, 15 (eyes, 4; verbal, 5; and motor, 6); body temperature $36.8^{\circ} \mathrm{C}$; pulse 98 beats $/ \mathrm{min}$; blood pressure 125/80 $\mathrm{mm} \mathrm{Hg}$; respiration $26 / \mathrm{min}$; and saturation of peripheral oxygen, $96 \%$ with $3 \mathrm{~L} / \mathrm{min}$ oxygen through the nasal cannula. Initial labs showed WBC $10.5 \mathrm{~g} / \mathrm{L}, \mathrm{RBC} 6 \mathrm{~T} / \mathrm{L}$, hemoglobin $128 \mathrm{~g} / \mathrm{L}$, PLT $37 \mathrm{~g} / \mathrm{L}$, total bilirubin $33.9 \mu \mathrm{mol} / \mathrm{L}$, albumin $27.8 \mathrm{~g} / \mathrm{L}$, AST $815 \mathrm{IU} / \mathrm{L}$, ALT 1332 IU/L, prothrombin time (INR) 2.28, APTT time (s) 33.8, D-Dimer 15,811 ng/mL, Fibrinogen $2.79 \mathrm{~g} / \mathrm{L}$. Cirrhosis workup for chronic liver disease was negative, including viral and autoimmune etiologies. Abdominal ultrasound confirmed the presence of ascites, and a diagnostic paracentesis revealed a serum ascites protein $15.2 \mathrm{~g} / \mathrm{L}$, Rivalta negative, and no evidence of spontaneous bacterial peritonitis. Abdominal computer tomography demonstrated hepatomegaly with the absence of blood in the HV whereas the portal vein was patent at this time. CT imaging also showed enlarged caudate lobe, hepatic parenchymal perfusion disorders, plenty of abdominal fluid, and bilateral pleural fluid (Fig. 1). Based on this information, BCS was suspected. Further workup, including JAK2 mutation, Factor V Leiden, antiphospholipid antibody syndrome, was negative despite the significant decrease of antithrombin III, protein S, and protein C (Table 1). The patient was initiated on $80 \mathrm{mg}$ low molecular weight heparin subcutaneously per day, simultaneously, underwent paracentesis for ascites and thoracentesis for pleural effusion. However, the patient grew increasingly abdominal distension with simultaneous continuing to have significant ascites and began to display asterixis. Within $48 \mathrm{~h}$, the patient developed hepatic encephalopathy stage II with blood ammonia level was $91 \mu$ /dL (normal range, 20-80), INR 2.74. Interdisciplinary consultation on the patient for urgent shunt placement was progressed because emergency orthotopic liver transplantation was unfeasible due to a lack of liver donor as well as financial constraints. Angiography was performed and demonstrated no thrombosis of the IVC. There was neither

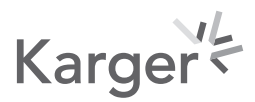



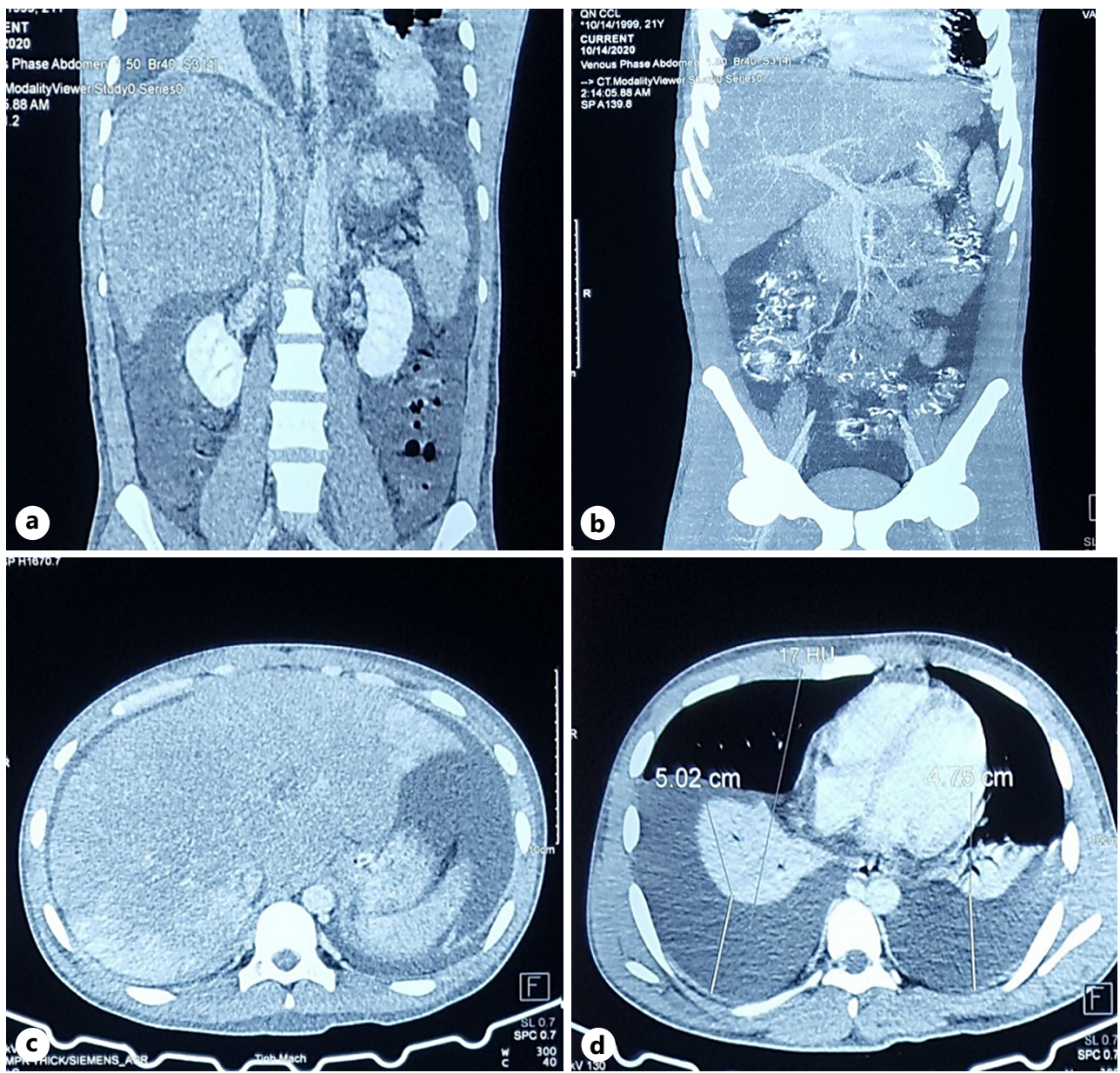

Fig. 1. a Contrast-enhanced abdominal CT finding CT image shows hepatomegaly with diffuse patchy enhancement due to hepatic congestion and necrosis. b The HVs are not identified as obstruction of flow prevents enhancement with intravenous contrast whilst the portal vein system is patent. c Contrast-enhanced abdominal CT finding CT image shows hepatomegaly with diffuse patchy enhancement due to hepatic congestion and necrosis. d Plenty of abdominal fluid, bilateral pleural fluid. HV, hepatic vein.

a normal-appearing HV nor any collateral veins to the IVC. In this case, the HV is entirely occluded and inaccessible to either recanalization or the creation of a TIPS. We decided to employ a DIPS without intravascular ultrasound (IVUS). After accessing the right transjugular, the liver was probed with the puncture needle from the IVC just below the origin of the HV. The guiding needle (Colapinto) was advanced through the liver parenchyma and rotated until an accessed right branch of the portal vein was verified by contrast injection. A guidewire and catheter were then put into the portal vein and a portal venogram illustrated the flow of hepatic parenchyma. The intrahepatic tract was dilated using an Evercross 8.0 dilatation balloon. The 10-French sheath was then passed through the intrahepatic tract to the portal vein. Two $9 \mathrm{~mm} \times 8 \mathrm{~cm}$ Protégé stents were placed spanning from the portal vein to the IVC on the first day of treatment (Fig. 2). Post-stenting venogram demonstrated excellent flow through the stented segment. After that patient's mentation began to improve and INR trended down. The daily volume of pleural effusion was significantly decreased; thus, his dyspnea was improved. On the 9th day, imaging of abdominal CT-scanner showed recurrent HV thrombosis occurred and trans-shunt thrombectomy whilst he has continued on low molecular weight 
Case Reports in Gastroenterology

Table 1. Admission laboratory results

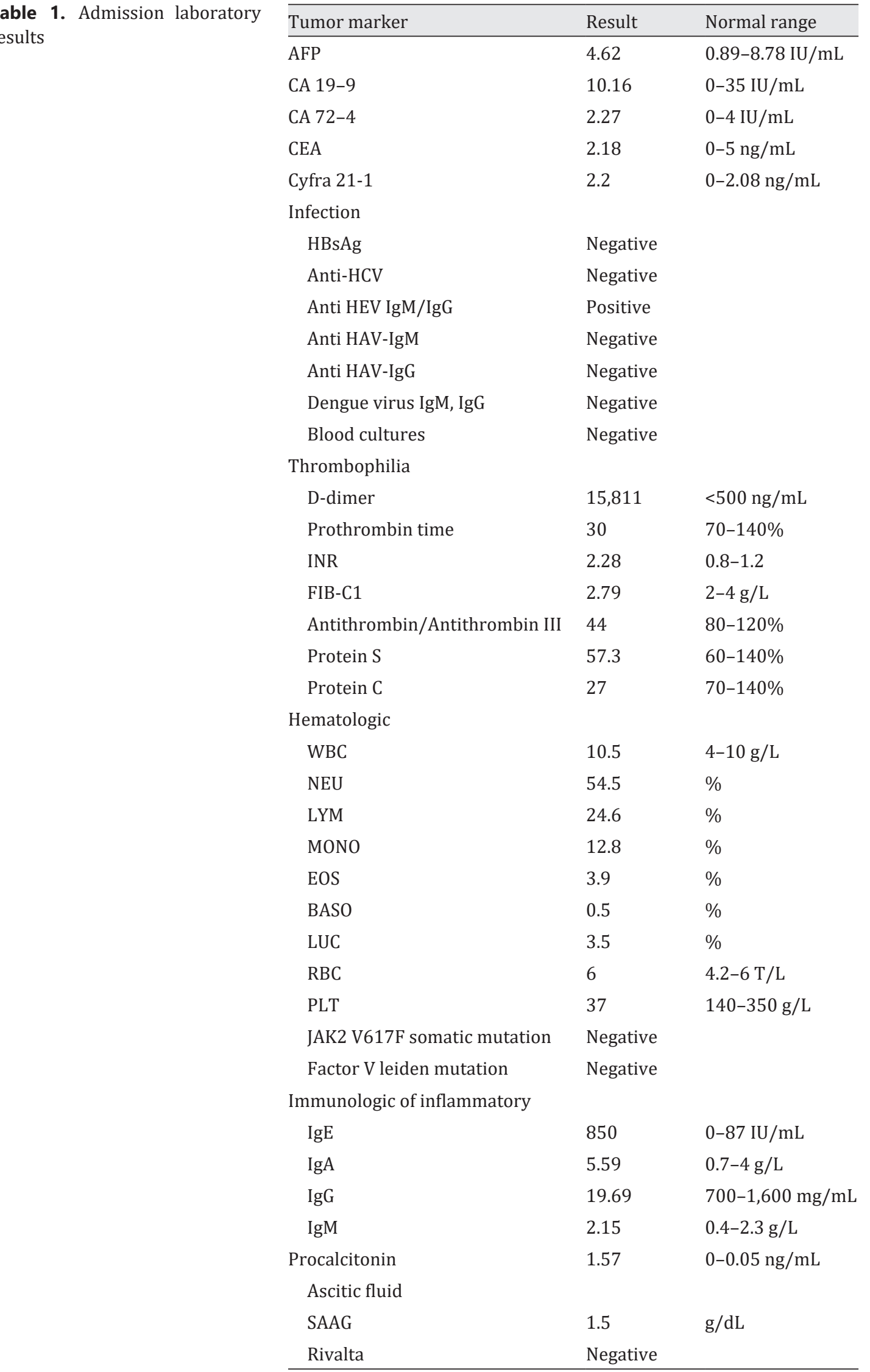



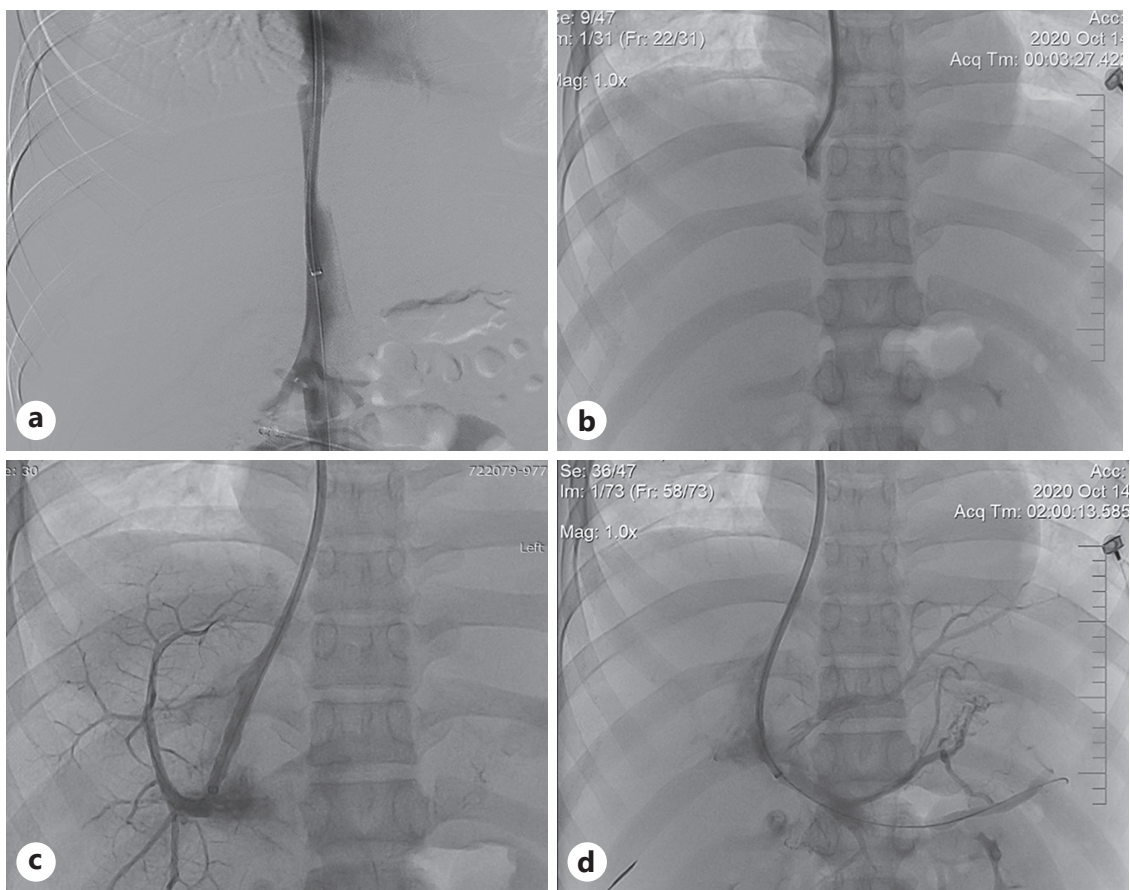

Fig. 2. a Portovenogram performed during DIPS procedure depicting the absence of the HV and no collateral veins to the IVC. HV, hepatic vein.b The puncture needle from the IVC just below the origin of the HV. HV, hepatic vein.c Access right branch of the portal vein verified by contrast injection. $\mathbf{d}$ Portal venography prior to creation and stenting of the DIPS. e Dilation of the intrahepatic tract. $\mathbf{f}$ Unsubtracted post-DIPS and stenting portal venography. HV, hepatic vein; IVC, inferior vena cava; DIPS, direct intrahepatic portosystemic shunt.

heparin; therefore, he was transitioned to oral rivaroxaban for long-term anticoagulation therapy. After postprocedure for 2 weeks, his mentation returned to normal, his abdominal distention resolved. The liver function of the patient was markedly recovered and discharged. At 3-month follow-up, the patient has returned to work with normal liver function test, exception for PLT 127 $\mathrm{g} / \mathrm{L}$ and INR 1.3. His DIPS is patent by ultrasound and CT-scanner of the abdomen (Fig. 3).

\section{Discussion and Conclusion}

BCS is characterized by hepatic venous outflow tract obstruction, regardless of the level or mechanism of obstruction [1]. According to the duration of the disease, BCS may be fulminant/acute, sub-acute, or chronic [2]. Despite the BCS is the most often sub-acute and chronic, however, there was about $1-2 \%$ of the cases of BCS with the manifestation of fulminant hepatic failure that is associated with very high mortality. If untreated, $70 \%$ of patients die within 1 year and 90\% within 3 years, usually from complications of liver cirrhosis [3]. Patients with the fulminant form present with hepatic encephalopathy within 8 weeks after the appearance of jaundice [4]. Symptoms of fulminant/acute BCS include intractable ascites, hepatic necrosis without the reveal of venous collaterals, and acute elevation of serum aminotransferase ( $>5$ times the upper limit of the normal range). The prognosis of fulminant or acute BCS without early diagnosis and intervention is poor. The severity of the disease is evaluated by the Rotterdam score based on the calculated score [5]:

Score $=1.27 \times$ encephalopathy $+1.04 \times$ ascites $+0.72 \times$ INR $+0.004 \times$ bilirubin 
Fig. 3. The shunt placed in patient remain patent to date.
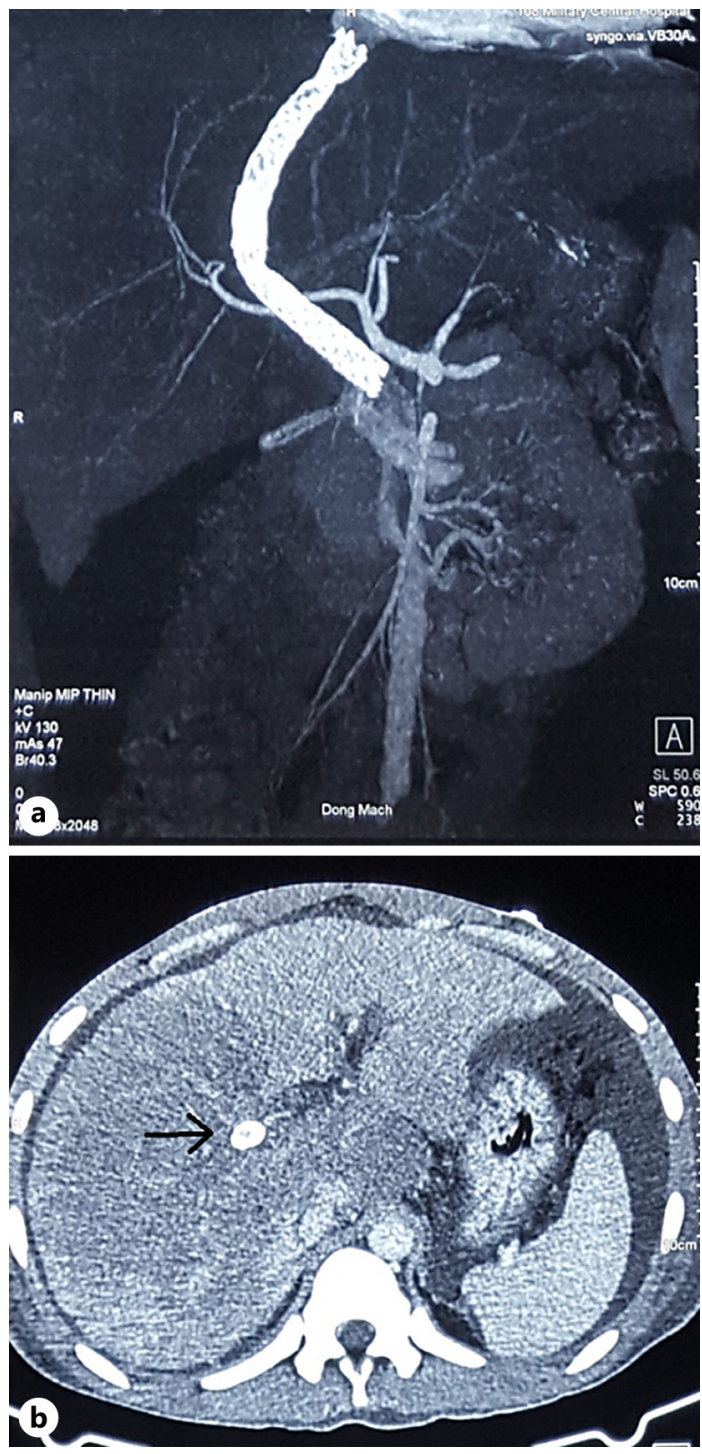

Encephalopathy and ascites are scored as either 0 (absent) or 1 (present), prothrombin time as 0 (INR $\leq 2.3$ ) or 1 (INR >2.3), and bilirubin in $\mu \mathrm{mol} / \mathrm{L}$. Class I scores between 0 and 1.1, class II scores between 1.1 and 1.5, and class III scores 1.5 and higher with 5-year survival rates of 89,74 , and $42 \%$, respectively. In the present case, the initial Rotterdam score of 1.90 , increased to 3.16 for $48 \mathrm{~h}$ later. Hence, the patient arrived at our hospital with liver function decreased severely. In addition to the use of prompt anticoagulation with low molecular weight heparin, he has indicated urgent intervention.

The goal of the endovascular intervention in BCS was to relieve hepatic congestion either through recanalization or the creation of a bypass. The least invasive interventional treatment is recanalization of HVs with and without stenting. Angioplasty was utilized commonly in Asia with good long-term outcomes since most of the BCS Asian population is associated with suprahepatic stenosis of the IVC [6]. By contrast, the pathophysiology of BCS in Western countries, hypercoagulable states often lead to complete HV occlusion; thus, the TIPS is regularly employed. Liver transplantation is only considered when endovascular procedures fail to control symptoms $[7,8]$. Despite TIPS has been shown a higher technical 
success rate in pediatric BCS patients than the angioplasty and HV stenting [9], in the adult population, no randomized controlled trials are comparing these interventional procedures. However, there are several retrospective evidence suggesting that TIPS may improve survival in BCS patients who fail to respond to medical therapy with high technical success rates of $93 \%$ and the 1-year and 5-year transplantation-free survival rate of 93 and $74 \%$, respectively $[10,11]$.

In some case, the TIPS procedure is not inaccessible because the $\mathrm{HV}$ is entirely occluded and unable to catheterize the HV. In such instances, DIPS is considered a viable alternative technique that can decompress the liver in these patients. Concerning the technique, the DIPS is the use of the caudate lobe as the parenchymal tract to create between the IVC and the right branch of the portal vein guiding IVUS [12]. Nevertheless, the demerit of the procedure is that it requires special equipment (IVUS) and is therefore expensive. Several interventionists modified this technique and used transabdominal ultrasound guidance to percutaneously insert a needle into a portal venous branch and subsequently directly into the IVC. Some cases and small series had chosen DIPS as a viable option for patients with acute and hyperacute BCS.

In reality, the proportion of acute BCS, especially, fulminant BCS, is a rare entity in Vietnam, and the treatment of BCS by DIPS does not have a report. Therefore, this is the first clinical case that has been performed DIPS procedure in our country. This case did not use ultrasound guidance to generate the DIPS due to the lack of IVUS with other devices. As aforementioned, the patient was in critical condition by fulminant liver failure and unfeasible transplant. DIPS is an alternative option for this case after TIPS failed. The most difficult part of this procedure is identifying and gaining access to the portal vein during fluoroscopy because we only utilized 2-dimensional images (hepatic venography) and the pathophysiology of BCS. However, we performed successfully DIPS within $150 \mathrm{~min}$. The patient was treated with rivaroxaban for long-term anticoagulation therapy. After 3 months of follow-up, the patient is in good health with normal liver function.

There is the first patient with fulminant BCS to treat by DIPS procedure in Vietnam. Our case provides evidence that DIPS is a viable alternative technique to TIPS that be performed successfully and safely in a patient with fulminant hepatic failure from BCS. Last but not least, long-term anticoagulation is needed to prevent Budd-Chiari recurrence and DIPS occlusion in these patients.

\section{Acknowledgements}

This manuscript does not include any non-author contributors to acknowledge.

\section{Statement of Ethics}

Authors' institution, the Institutional Review Board of 108 Military Central Hospital, does not require ethical approval for publication of a case report. Written informed consent was obtained from the patient for publication of this case report and any accompanying images.

\section{Conflict of Interest Statement}

The authors declare no conflict of interest about this work.

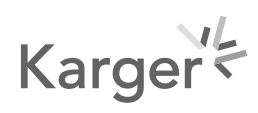


Nguyen et al.: DIPS for Fulminant BCS

\section{Funding Sources}

There are no funding sources for this case report.

\section{Author Contributions}

T.V.N. and H.D.D. collected the data. T.V.N. and H.Q.D. did the literature reviews. T.V.N. prepared the first manuscript. T.T.N., H.Q.D., and H.D.D. involved in correction of the manuscript. All authors (T.V.N., T.T.N., H.D.D., and H.Q.D.) have read and approved the final manuscript.

\section{Data Availability Statement}

All data generated or analyzed during this study are included in this article. Further enquiries can be directed to the corresponding author.

\section{References}

1 Mukund A, Sarin SK. Budd-Chiari syndrome: a focussed and collaborative approach. Hepatol Int. 2018;12(6): 483-6.

2 Van Wettere M, Bruno O, Rautou PE, Vilgrain V, Ronot M. Diagnosis of Budd-Chiari syndrome. Abdom Radiol. 2018;43(8):1896-907.

3 Fu Y, Sun YL, Ma XX, Xu PQ, Feng LS, Tang Z, et al. Necessity and indications of invasive treatment for BuddChiari syndrome. Hepatobiliary Pancreat Dis Int. 2011;10(3):254-60.

4 Menon KV, Shah V, Kamath PS. The Budd-Chiari syndrome. N Engl J Med. 2004;350(6):578-85.

5 Darwish Murad S, Valla DC, de Groen PC, Zeitoun G, Hopmans JA, Haagsma EB, et al. Determinants of survival and the effect of portosystemic shunting in patients with Budd-Chiari syndrome. Hepatology. 2004;39(2): $500-8$.

6 Qi XS, Ren WR, Fan DM, Han GH. Selection of treatment modalities for Budd-Chiari Syndrome in China: a preliminary survey of published literature. World J Gastroenterol. 2014;20(30):10628-36.

7 Khan F, Armstrong MJ, Mehrzad H, Chen F, Neil D, Brown R, et al. Review article: a multidisciplinary approach to the diagnosis and management of Budd-Chiari syndrome. Aliment Pharmacol Ther. 2019;49(7):840-63.

8 Mancuso A. Budd-Chiari syndrome management: lights and shadows. World J Hepatol. 2011;3(10):262-4.

9 Sharma VK, Ranade PR, Marar S, Nabi F, Nagral A. Long-term clinical outcome of Budd-Chiari syndrome in children after radiological intervention. Eur J Gastroenterol Hepatol. 2016;28(5):567-75.

10 Garcia-Pagán JC, Heydtmann M, Raffa S, Plessier A, Murad S, Fabris F, et al. TIPS for Budd-Chiari syndrome: long-term results and prognostics factors in 124 patients. Gastroenterology. 2008;135(3):808-15.

11 Rössle M, Olschewski M, Siegerstetter V, Berger E, Kurz K, Grandt D. The Budd-Chiari syndrome: outcome after treatment with the transjugular intrahepatic portosystemic shunt. Surgery. 2004;135(4):394-403.

12 Petersen BD, Clark TW. Direct intrahepatic portocaval shunt. Tech Vasc Interv Radiol. 2008;11(4):230-4.

\section{Karger' ${ }^{\prime \prime}$}

